\title{
The recognition of chaos in host-pathogen response
}

\author{
Kumar Selvarajoo ${ }^{1,2 *}$ \\ 1 Institute for Advanced Biosciences, Keio University, Tsuruoka, Yamagata, Japan \\ 2 Systems Biology Program, Graduate School of Media and Governance, Keio University, Fujisawa, Japan \\ ${ }^{*}$ Correspondence: kumar@ttck.keio.ac.jp
}

The immune system is central to living organisms' survival. The fascinating accuracy and repeatability of specific cellular signaling process triggered by an invading agent to the host cells has received immense attention in biological research over the last decades. For microorganisms, such as E. coli and yeast, the formation of layered structures, such as biofilms, enhances their survival capacity in the host through cooperative behavior. Although this fascinating self-organizing feature has intrigued scientists ever since the Darwin's era, recently, there has been great interest in the area of human health through the observation of resistance and persistence mechanisms of infectious microbes to anti-microbial agents (Smith and Romesberg, 2007; Høiby et al., 2010).

The formation of bacterial films is causing fatal consequences to those who are especially immuno-compromised. It is also a nuisance for those who require surgical insertions, where biofilms have been noticed to flourish around the implanted devices. Furthermore, the ability of infectious microbes to evade host immune cell attacks is also scientifically intriguing. Thus, numerous efforts are underway worldwide to develop vaccines and other drugs that would prevent or slow the formation of biofilm. However, presently, little is known about host's poor response mechanisms to bacterial biofilms. It will be important to understand the host-biofilm interactions from a systemic perspective so that effective holistic strategies could be investigated to suppress and kill biofilms.

Traditionally, and still common practice, intracellular signal transduction processes of host-pathogen interactions are studied through wet experimental data on population-wide average cell approach. That is, pathogen infected living cells are lysed, a selected number of key proinflammatory molecules, such as MAP kinases and $\mathrm{NF}-\kappa \mathrm{B}$, are purified and extracted over cell population. In such a scenario, variability in the level of measured components can be thought to be undesirable as they will interfere with reliable deterministic immune processes. More recent works generate high-throughput data at the transcriptomeand proteome-wide expression levels. The systemic analyses of these data also represent population-wide averages. If biological processes are governed by well-defined deterministic processes alone, how then is a single fertilized cell able to differentiate into multi-cell types in higher organisms and how do diseases occur and progress?

Modern techniques on single-cell and molecule resolution reveal that gene and protein expressions between cells of an otherwise identical group are stochastic in time and clonal population of cells of specific cell type display heterogeneity in the abundance of a given protein per cell at any measured time (Brock et al., 2009; Eldar and Elowitz, 2010). Today, combinatorially, stochasticity and heterogeneity are considered as biological noise and are essential for generating phenotypic variation, cell fate decisions, and amplification of molecular signals. So, how does the effect of biological noise affect our understanding of host-pathogen interactions?

In physics, the term "chaos" represents seemly random or unpredictable phenomenon, that is actually governed by deterministic process and often expressed with simple rules (Lorenz, 1995). Accepting the fact that biology is a chaotic living system, it becomes relevant for the existence of biological noise and deterministic process at the same time. For example, for cellular differentiation process, we require a single-cell origin to diversify its lineage on a deterministic process in time (Figure 1A). This event is in analogy to the simple example of a chaotic process represented by a ski slope with bumps (Mogul skiing, Figure 1B insert). Here, the gravitational force drive the skier to the finish point and the bumps create a landscape to develop error or noise, that could change the lineage of each trajectory (attractor) to distinct pathways on different attempts
(Figure 1B). Considering such concepts brings our attention to the importance of noise in biology and the "management" of such noise could benefit in the control of host-pathogen interactions.

Biofilms are emergent structures that stand firm on a stable attractor and overcome the original programmed attack mechanisms of the host cells. Hence, there is a need to switch from the current unsuccessful host-pathogen response to a reprogrammed approach that will become sensitive to the attack and destroy the cooperative organization. Since single-cell analysis demonstrates biological noise, to overcome the resistance and persistence mechanisms for biofilm survival require the host cells to utilize single-cell noise to switch the pathogen's attractor state, just as shown in the changing fate of ski trajectories to error (Figure 1B). The main challenge is in the determination of what biological noise that requires manipulation. For the iPS cells, we now fully appreciate the socalled Yamanaka factors (Oct3/4, Sox2, Klf4, $\mathrm{c}-\mathrm{Myc})$ are crucial for changing attractor states of cell differentiation (Takahashi and Yamanaka, 2006). The same could also be possible for dealing with biofilm management and future research could focus on the elucidation of such potential molecular factors.

In my opinion, there is a need to distinguish the differences at the single-cell and population-wide scales in biology, so as to allow us to reveal the mechanistic causes for ordered response emerging from disordered response, such as the biofilm formation. This requires us to treat single-cell microscopic and population-wide macroscopic dynamics distinctly (Selvarajoo, 2011), just like quantum and classical theories are used to interpret physical properties and behaviors at different scales in physics. Thus, in biology, there is a need to further develop and introduce novel mathematical theories and to identify governing principles. Understanding the causes for ordered response using non-linear approaches and 
A

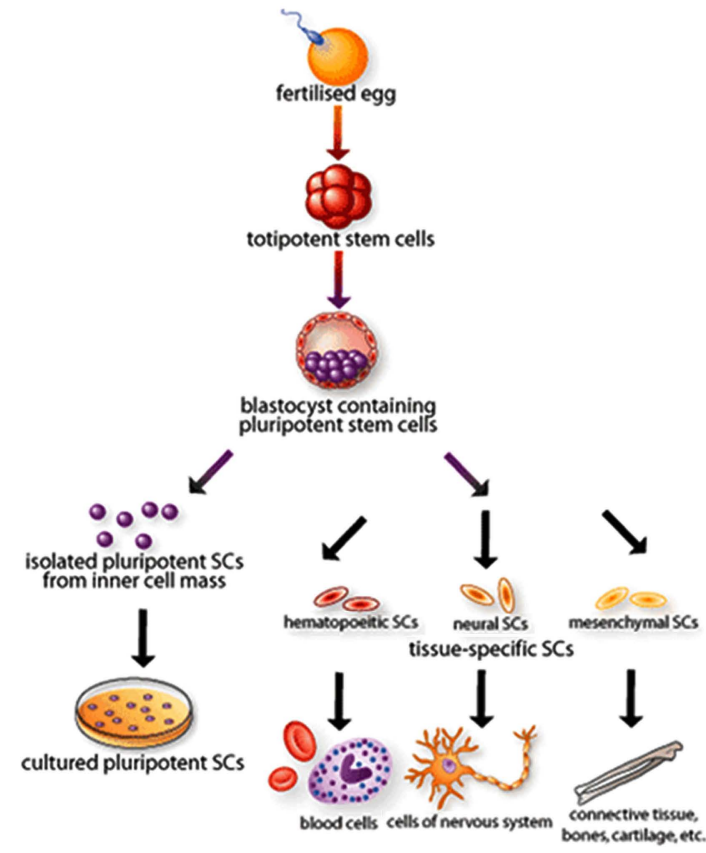

B

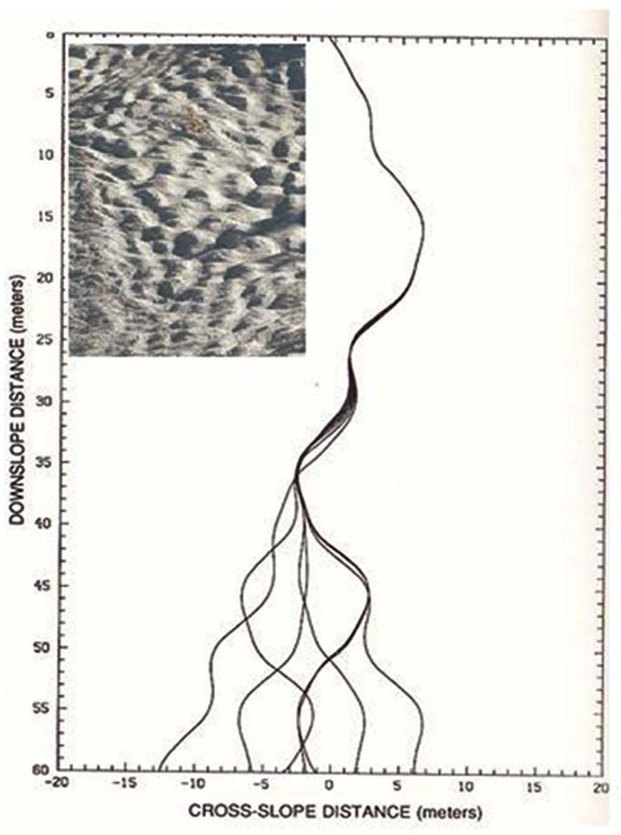

FIGURE 1 | Cell differentiation can be seen as a deterministic chaotic process. (A) Schematic of mammalian cell differentiation process, obtained from Chaudry (2004). (B) Diverging endpoints of seven boarders' trajectories with minute (1 mm) changes in course maneuver at a ski slope with moguls (insert), adapted from Lorenz (1995).

noise analyses could fundamentally provide crucial cues for the successful management of our immune response to eradicate the emergent self-organizing structures of invading pathogens.

\section{ACKNOWLEDGMENTS}

I appreciate the research fund of Yamagata Prefecture and Tsuruoka city, Japan.

\section{REFERENCES}

Brock, A., Chang, H., and Huang, S. (2009). Non-genetic heterogeneity-a mutation-independent driving force for the somatic evolution of tumours. Nat. Rev. Genet. $10,336-342$.
Chaudry, A. (2004). Stem Cell Bioengineering. Available at: http://www.scq.ubc.ca/stem-cell-bioengineering/

Eldar, A., and Elowitz, M. B. (2010). Functional roles for noise in genetic circuits. Nature 467, 167-173.

Høiby, N., Bjarnsholt, T., Givskov, M., Molin, S., and Ciofu, O. (2010). Antibiotic resistance of bacterial biofilms. Int. J. Antimicrob. Agents 35, 322-332.

Lorenz, E. (1995). The Essence of Chaos (The Jessie and John Danz Lecture Series). Seattle: University of Washington Press.

Selvarajoo, K. (2011). Macroscopic law of conservation revealed in the population dynamics of toll-like receptor signaling. Cell Commun. Signal. 9, 9.

Smith, P. A., and Romesberg, F.E. (2007). Combating bacteria and drug resistance by inhibiting mechanisms of persistence and adaptation. Nat. Chem. Biol. 3, 549-556.
Takahashi, K., and Yamanaka, S. (2006). Induction of pluripotent stem cells from mouse embryonic and adult fibroblast cultures by defined factors. Cell 126, 663-676.

Received: 12 December 2011; accepted: 10 January 2012; published online: 26 January 2012.

Citation: Selvarajoo K (2012) The recognition of chaos in host-pathogen response. Front. Physio. 3:7. doi: 10.3389/ fphys.2012.00007

This article was submitted to Frontiers in Systems Biology, a specialty of Frontiers in Physiology.

Copyright $(\odot 2012$ Selvarajoo. This is an open-access article distributed under the terms of the Creative Commons Attribution Non Commercial License, which permits non-commercial use, distribution, and reproduction in other forums, provided the original authors and source are credited. 